\title{
Performance Analysis of the Sliding Mode Control for Automated Vehicle Path Tracking at Low Adhesion Surfaces
}

\author{
Ilya Kulikov ${ }^{1, a}$ and Ivan Ulchenko ${ }^{1}$ \\ ${ }^{1}$ State Research Centre "NAMI", Moscow, Russian Federation
}

\begin{abstract}
The article analyses prospects of using a type of robust controllers called relay regulators for automation of vehicle lateral motion. The operation of these regulators in so-called sliding modes is considered along with the "chattering" problem caused by deviations from the "ideal" sliding mode inevitable in actual implementations. For the analysis of vehicle motion, a mathematical model was elaborated, which calculates vehicle dynamics taking into account non-linear tire-road adhesion characteristics. In the conducted study, emphasis was put on low adhesion surfaces, which can be considered as the most difficult case for automatic lateral control of a vehicle. In order to implement automated path tracking within the model, two relay regulators were elaborated differing from one another in the order of dynamics. A comparative study of these regulators was conducted by means of simulations. The regulator that had shown best performance was then tested for robustness by means of modeling, in which maneuvers on snow, ice and a mixed surface were simulated.
\end{abstract}

\section{Introduction}

Development of automated driving functions for vehicles constitutes a complex task, since the controlled object is non-linear, has parameter uncertainties, and operates under arbitrary conditions. Automated maneuvering at low adhesion or mixed-adhesion surfaces can be considered as most difficult control task requiring of the automated driving functions involved to be highly adaptive. The objective of this work is to develop an adaptive (robust) controller solving this control task.

In the literature, one can find at least five controller types used (or offered to be used) in automated driving functions, namely, linear-quadratic regulators (LQR), model predictive regulators (MPC), fuzzy controllers, PID (PI, PD) regulators with gain scheduling, and relay regulators operating in sliding modes.

The first two regulator types are derived from models of controlled plants. In application to automated vehicle driving, these regulators, being accompanied by rather advanced and complex observers of vehicle dynamic parameters, show good performance even in emergency maneuvers at low adhesion surfaces [1,2]. LQR and MPC require parameters of the controlled plant to be known (identified). The stability criterion of these regulators is observability of matrices, which contain model parameters; however, this observability may substantially diminish in complicated driving conditions such as maneuvering at mixed surfaces. The second issue is linearity of LQR and MPC. Since the vehicle is nonlinear object, using these regulators for its control implies linearization of the model at each time step of the controller. The third issue is high computational burden imposed by solving of regulator equations. It is admitted that existing processing units available for vehicles are not able to perform these calculations when vehicle speed increases beyond certain values.

Using conventional PID regulators for automated driving is only possible with scheduled gains. This makes regulators non-linear allowing them to handle vehicle dynamics and encompass various driving conditions. Examples presented in the literature demonstrate a good performance of such regulators in various (yet limited) tasks $[3,4]$. It is clear that "schedules" are expected to be multidimensional lookup tables being functions of variables, which characterize both vehicle parameters and driving conditions (the latter are supposed to be observable). A considerable number of simulations and field-testing are needed to populate and tune those tables. However, any internal and ambient factors lying outside the range of predefined tables will question stability and adaptiveness of the regulator.

As an alternative to conventional and optimal regulators, fuzzy controllers are making their way into vehicle active safety systems and driving automation applications. However, the fuzzy approach may be problematic when it comes to the general task of driving control rather than its particular cases such as lane keeping [5] or low-speed maneuvering [6]. The first issue is that increasing of control task complexity implies expanding sets of fuzzy rules and terms. This, in turn, entails higher efforts to develop a controller and increases computational burden that may even make one resort to using separate processing units performing fuzzy

\footnotetext{
${ }^{a}$ Corresponding author: i.kulikov.mami@gmail.com
} 
operations. The second issue is that the general task of driving control makes it difficult to encompass the variety of possible driving conditions and vehicle parameters by a limited set of fuzzy rules and terms. One should also take into account that the foundation of fuzzy controllers is an expert knowledge, which becomes a decisive factor of controller's robustness. The question is if this knowledge, even when provided by qualified experts, can be adequate for entire variety of possible driving situations including emergency maneuvers, when vehicle behavior becomes strongly non-linear and difficult to handle.

Relay regulators [7, 8] are free from the described shortcomings of model-based adaptive controllers (although optimal relay regulators can also be based on models) and augmented conventional regulators. Relay regulators are non-linear "by nature", which makes them suitable for control of non-linear plants with no linearizing assumptions. In the "ideal" sliding mode (explained below), both structure and operation of relay regulators only depend on the tracking error and its derivatives and are insensitive to plant's parameter uncertainties or disturbances. The structure of a relay regulator is simple, thus, not imposing high computational burden upon processing units.

The major problem of relay regulators is so called chattering [8, 9] - high frequency, sawtooth-form oscillations induced by switching of the relay element with finite frequency, which is inevitable in an actual controller having finite time step. This is a malicious phenomenon, which causes noise and vibrations and even can lead to damaging of control mechanisms and some parts of the controlled object. In the literature, a few methods are proposed to reduce chattering, namely, the boundary layer approach [10], the twister and supertwister methods [11], using of compensating state observers [8], and non-linear adjustment of the relay gain [8]. Yet another method is use of higher order sliding modes [11]. It is considered a perspective one; however, it makes regulator dynamics more complex, since it implies high orders of derivatives and integrals involved. This may entail diminishing of regulator accuracy and stability. On the other hand, these (possible) adverse effects depend on specifics of the given plant and its dynamics and only can be estimated through appropriate studying.

Despite their peculiarities and difficulties stemming from the chattering problem, sliding mode regulators are recognized as perspective robust controllers. A number of works in the field of vehicle dynamics and automated driving are dedicated to these regulators [12, 13]. A preliminary analysis suggests that the higher order sliding modes can be suitable for vehicle automated driving on the condition of providing both chattering suppression and stability of operation. This work describes an investigation of this problem putting emphasis on maneuvers performed at different low adhesion surfaces implying automated driving functions to be adaptive to ambient conditions.

A mathematical model of vehicle dynamics was elaborated in order to conduct said study. Adequacy and accuracy of the model were estimated through a comparison with experimental data obtained from tests, which were performed at actual low adhesion surfaces. An automatic path-tracking function was implemented within the model. The sequel of the article describes these models and the results of the conducted study.

The paper is organized in three main sections. Section 2 describes the developed model of vehicle dynamics. In section 3, elaboration and model analysis of the pathtracking controller are presented including a comparison of different designs of sliding mode regulators. The final section offers concluding remarks and outlines the future work.

\section{Model of vehicle dynamics}

A detailed description of the vehicle dynamics model used in this study can be found in [14], while in this article, it is only presented briefly. The model constitutes a system of six lumped masses, namely, the vehicle mass whose motion, projected onto the road, is considered planar, the sprung mass performing angular motion (i.e. body roll) in the lateral plane of the vehicle, and four rotating masses of the wheels. The wheel normal forces are calculated from the static equilibrium of the vehicle in both longitudinal and transversal planes.

The following coordinate systems are introduced within the model: the road-bound system, the vehiclebound system (the variables belonging to it are denoted with indices containing " $v$ "), and the wheel-bound systems (corresponding variables are provided with indices containing " $w$ "). Variables related to individual wheels are also provided with indices $i j$, where $i$ stands for the axis number, and $j$ denotes number of the wheel associated with $i$-th axis. The model of vehicle dynamics has the form of the following equation system:

$$
\left\{\begin{array}{l}
m \cdot\left(\dot{V}_{C o G, x-v}-\omega_{z} \cdot V_{C o G, y-v}\right)=\sum_{i=1}^{2} \sum_{j=1}^{2} R_{x-v . i j}-F_{w, x} \\
m \cdot\left(\dot{V}_{C o G, y-v}+\omega_{z} \cdot V_{C o G, x-v}\right)=\sum_{i=1}^{2} \sum_{j=1}^{2} R_{y-v . i j} \\
J_{z} \cdot \ddot{\Psi}_{z}=\mathcal{J}_{z} \cdot \dot{\omega}_{z}=\sum_{i=1}^{2} \sum_{j=1}^{2} M_{z-v . i j} \\
J_{x} \cdot \ddot{\varphi}=\sum_{i=1}^{2} \sum_{j=1}^{2}\left(R_{y-v . i j} \cdot h_{C o G}\right)-\varphi \sum_{i=1}^{2} c_{\varphi i}-\dot{\varphi} \sum_{i=1}^{2} d_{\varphi i}
\end{array}\right.
$$

where $m$ is the vehicle mass, $V_{C o G, x-v}$ and $V_{C o G, y-v}$ are the components of the vehicle velocity vector in the center of gravity (CoG) projected onto vehicle's longitudinal and lateral axes respectively, $\omega_{z}$ and $\psi_{z}$ are the vehicle yaw rate and yaw angle respectively, $R_{x-v . i j}$ and $R_{y-v i j}$ are the components of the tire force projected onto vehicle's longitudinal and lateral axes respectively, $M_{z-v . i j}$ is the yawing moment exerted by the wheel, $\mathcal{J}_{z}$ is the vehicle yaw inertia, $\mathcal{J}_{x}$ is the roll inertia of the sprung mass, $\varphi$ is the body roll angle, $h_{C o G}$ is the CoG height, $c_{\varphi i}$ and $d_{\varphi i}$ are the roll stiffness and damping of the suspension. 
In the simulations conducted in this work, the vehicle was maneuvering with no combined tire slip (i.e. slip, which consists of longitudinal and side components both having substantial magnitudes). In that case, the tire forces in the wheel-bound coordinate system and the tire aligning moment $\left(M_{z-w}\right)$ can be calculated (with some simplifying assumptions) by the following functions: $R_{x-w}=R_{z} \cdot \mu_{x}\left(S_{x}\right), R_{y-w}=R_{z} \cdot \mu_{y}(\alpha), M_{z-w}=R_{y-w}$. $t_{0}(\alpha)$. In these formulae, $\mu_{x}$ and $\mu_{y}$ are longitudinal and lateral tire-road adhesion coefficients respectively, $S_{x}$ is the longitudinal tire slip, $\alpha$ is the sideslip angle, and $t_{0}$ is the tire pneumatic trail.

The components of the tire slip are calculated by expressions, which include linear and angular velocities calculated by the equation system (1). These expressions are omitted here and can be found in [14]. For calculation of the adhesion coefficients and the pneumatic trail, an empirical tire model called the Magic Formula [15] was employed.

The model was verified for a passenger vehicle [14] (the gross mass $2550 \mathrm{~kg}$ ), which is studied in this work.

\section{Elaboration and model analysis of the path-tracking control}

The path-tracking regulator operating principle is to minimize deviation between the vehicle driving direction and the reference traveling path. Figure 1 shows a schematic used for calculation of this deviation. In order to make the regulator respond to changes in the required path in advance, the deviation is observed at the point lying ahead of the vehicle, on the extension of its longitudinal axis. The distance between the vehicle CoG and the observation point is denoted "x-obs". The required vehicle trajectory is defined by an array of coordinates, which is approximated by the linear piecewise method. Thus, each fragment of the trajectory is presented by a linear algebraic equation. A similar equation describes the line being normal to the vehicle longitudinal axis and intersecting it in the observation point. Combining equation of this orthogonal line with the equation of the trajectory's piece, which is nearest to the vehicle, allows obtaining the intersection point of these two lines and, consequently, yields the deviation $\Delta y$. Calculation of this deviation is based on rather simple geometry; however, the corresponding equations are bulky and therefore omitted here.

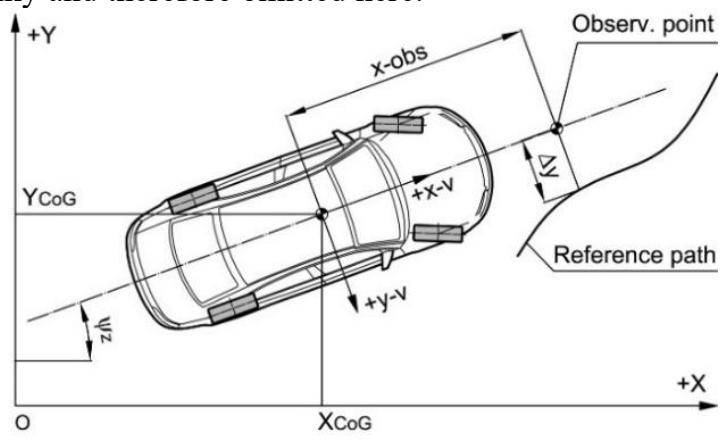

Figure 1. Schematic to calculate deviation of the vehicle from the reference path.
Implementation of a control system based on a relay regulator arises the question how to translate a relay signal into the command for an actuator. In the case of vehicle trajectory control, the latter constitutes a steering mechanism. For its automatic control, the steering angle command is required. It is obvious that this command should be a continuous signal. Moreover, neither steering speed nor angular acceleration can be discontinuous signals. This implies placing series of integrators between the relay signal and the steering angle command. However, increasing order of integration may entail degrading of regulator stability and tracking quality. Thus the number of integrators should be a trade-off between smoothness of the regulator output signal and the stability (and quality) of its operation. During the study, it was established that the minimum admissible order of integration is second, which implies relay switching to be at the level of angular acceleration signal. The mathematical description of the resulted regulator (hereafter called the Regulator-1) reads as follows:

$$
\ddot{\delta}_{S W}=\gamma \cdot \operatorname{sgn}(s) \text {. }
$$

where $\delta_{S W}$ is the steering wheel angle. Instead of $s g n$ function, a relay element may be employed. The integrators placed after the relay element have limits, which define the maximum steering speed (in this study, the permissible speed range was $\pm 300 \%$ s) and the maximum steering angle (was constrained by the range $\left.\pm 200^{\circ}\right)$.

In theory, the relay gain $\gamma$ should be infinite that would have resulted in infinite switching frequency yielding the "ideal" sliding mode. It is obvious that this cannot be implemented in actual applications; therefore, the value of gain is set finite yet sufficiently large. Studying the regulators described in this work allowed to conclude that increasing the gain up to $1 \mathrm{e} 5$ improves the regulator performance. Above that value, no changes were observed.

$s=f(\Delta y)$ is a function defining so-called hyperplane of the sliding mode or the sliding surface. In the "ideal" sliding mode, the phase trajectory of the controlled plant follows along this surface. In actual sliding modes, phase trajectories dwell in some vicinity of the sliding surface. For the Regulator-1, this surface was defined as follows:

$$
s=c_{1} \Delta y+c_{2} \Delta \dot{y}+c_{3} \Delta \ddot{y}
$$

where $c_{1}, c_{2}, c_{3}$ are tunable parameters defining the shape of the sliding surface.

For model analysis of the path-tracking regulators, different maneuvers were conducted, one of which implied driving along the test track shown in the uppermost graph in Figure 2. The three bends of the track have the following radii (from left to right): $35 \mathrm{~m}, 40 \mathrm{~m}$, and $40 \mathrm{~m}$. The vehicle was driving at a constant speed, which, at a given surface, required rather intensive steering in order to follow the trajectory with an acceptable accuracy. On the other hand, the speed was not high enough to bring the vehicle into a skid or drift, because this would have required special measures to maintain the vehicle yaw stability, which was beyond the scope of the study.

Figure 2 shows the modeling results with involvement of the Regulator-1. The graphs show the following 
variables (from top to bottom): vehicle trajectory, the output signal of the relay element, the steering angle signal. In these graphs, one can see that the regulator provides satisfactory accuracy of path tracking; however, the steering angle signal contains a pronounced chattering, which is undesirable for actual implementation of the control system within a vehicle.

For elimination of the chattering, the regulator order (i.e. the order of both input derivatives and output integrators) was increased by one. The resulted design is referred to as the Regulator-2. Higher orders of the derivatives and integrators entail questions of the noises at the regulator input and stability of its operation. The first question requires an additional investigation, which lies beyond the scope of the described study. The stability issue was investigated by simulations of the above maneuver. Figure 3 shows the simulation results for the same driving conditions as were used previously for the Regulator-1.
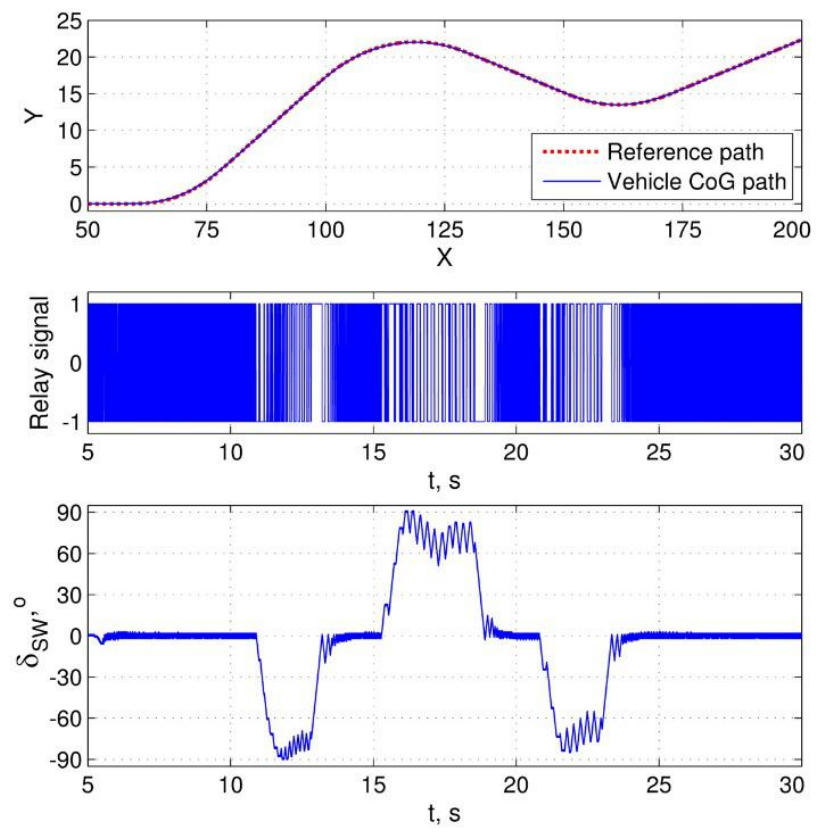

Figure 2. Simulation results for the test track covered with snow. Regulator-1. Vehicle speed $35 \mathrm{~km} / \mathrm{h}$.
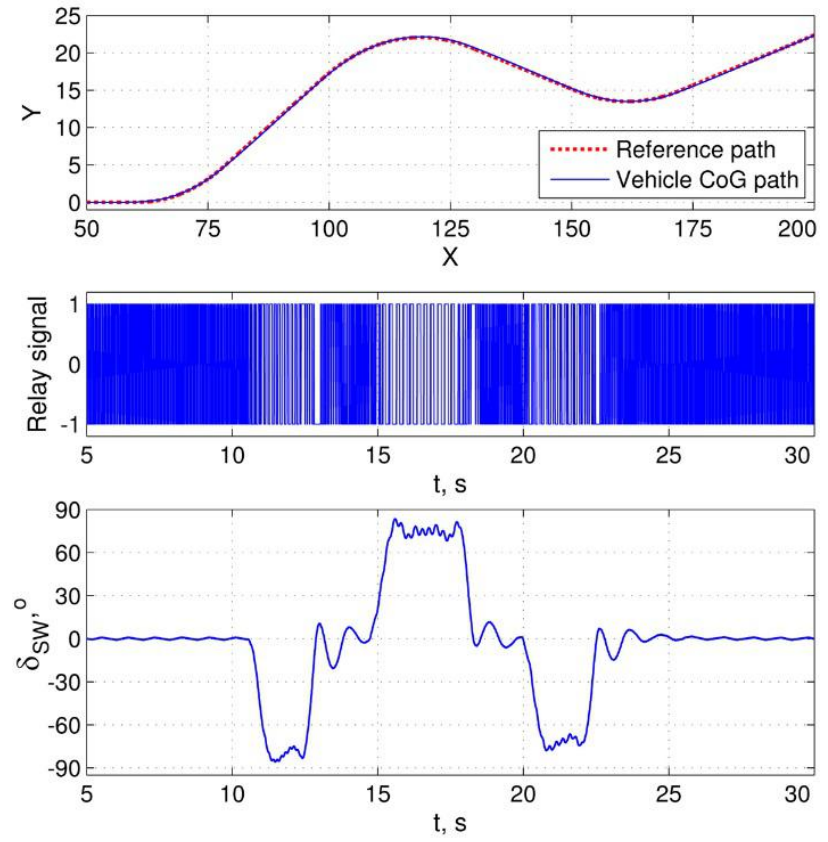

Figure 3. Simulation results for the test track covered with snow. Regulator-2. Vehicle speed $35 \mathrm{~km} / \mathrm{h}$.

From these graphs, one can make the following observations. Using of the Regulator-2 eliminates chattering from the output signal. In this aspect, the Regulator-2 makes a possible candidate for implementation within an actual system. Both the regulator operation and vehicle motion are stable. The path tracking accuracy degraded negligibly. Oscillatory behavior of steering transients has increased, which was expected due to higher integration order (i.e. increased phase shifting). However, the oscillations have relatively small amplitude and fade rapidly. Self-oscillations of smaller amplitude and higher frequency are observed when the mean value of steering angle is close to constant (see also Figure 4). Although this is not chattering, such oscillations of the steering wheel are undesirable. Therefore, in the sequel of the research, methods to damp these oscillations are to be found.

In order to investigate robustness of the sliding mode, i.e. regulator's behavior in differing and complicated driving conditions, the Regulator-2 was tested at icecovered track and at mixed surface imitating a snowy road with icy patches. The simulation results for both surface types are shown in Figures 4 and 5. In the trajectory graph for the mixed surface, the grey areas depict mentioned icy patches.

In the both cases, the regulator has provided stable operation and good tracking accuracy. At the mixed surface, deviations from the reference path are slightly higher than at the homogeneous snowy surface. At the icy surface, one can notice above-mentioned self-oscillations of the steering angle signal, while at the mixed surface there are no such oscillations due to absence of steady state steering. Note that in all the described simulations, the regulator parameters (i.e. gains) remained unchanged. 

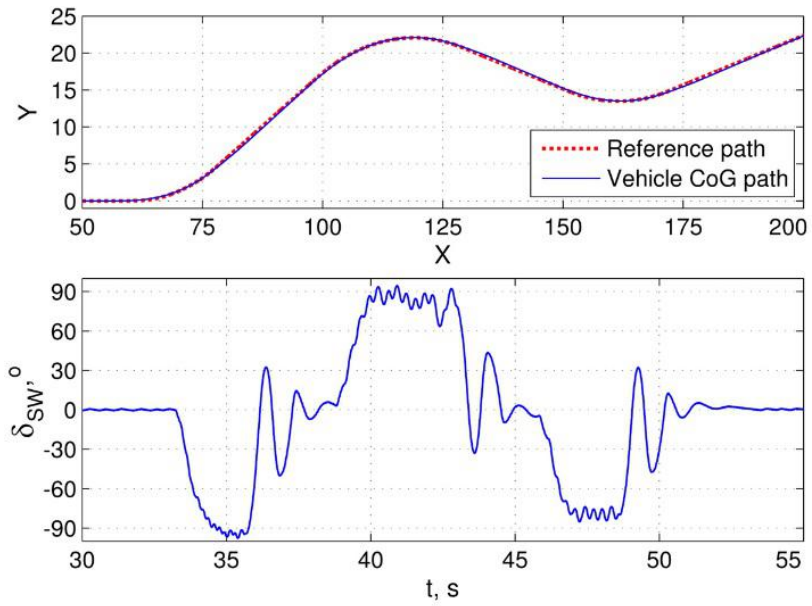

Figure 4. Simulation results for the test track covered with ice. Regulator-2. Vehicle speed $28 \mathrm{~km} / \mathrm{h}$.

Table 1 summarizes path-tracking performance of the elaborated regulators in the above maneuvers at different surfaces. Two parameters are used to characterize the tracking accuracy, namely, the mean square value (MSV, in meters) and the maximum value (Max., in meters) for the deviation of the $\mathrm{CoG}$ trajectory from the reference path (excluding the first straight-line part of the test track where no steering is required).
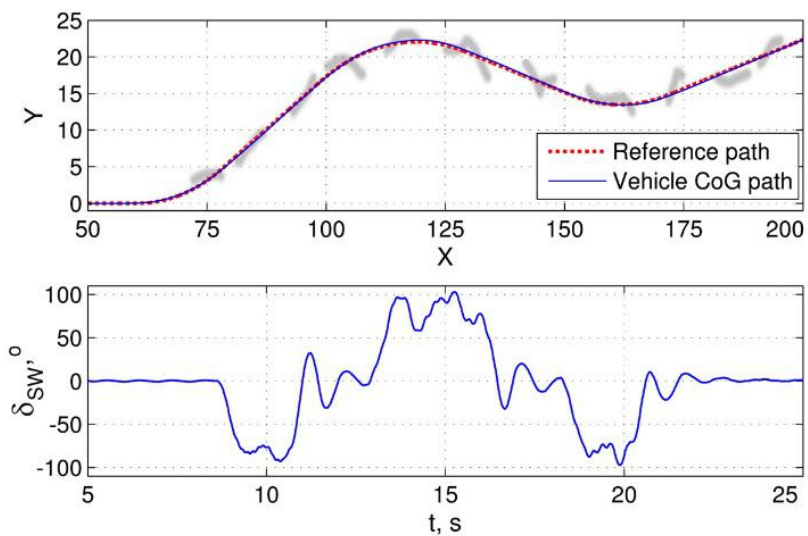

Figure 5. Simulation results for the test track with mixed surface (snow and ice). Regulator-2. Vehicle speed $35 \mathrm{~km} / \mathrm{h}$.

Table 1. Accuracy parameters of the path-tracking regulators at different surfaces.

\begin{tabular}{|l|c|l|c|c|c|c|}
\hline \multirow{2}{*}{ Reg.\# } & \multicolumn{2}{|c|}{ Snow } & \multicolumn{2}{c|}{ Ice } & \multicolumn{2}{c|}{ Mixed } \\
\cline { 2 - 7 } & MSV & Max. & MSV & Max. & MSV & Max. \\
\hline 1 & 0.05 & $<0.1$ & n/a & n/a & n/a & n/a \\
\hline 2 & 0.065 & $<$ & 0.13 & $<$ & 0.1 & $<0.2$ \\
& & 0.15 & & 0.28 & & \\
\hline
\end{tabular}

\section{Conclusions and future work}

The conducted study has shown that the sliding mode control, even non-ideal, provides a considerable robustness. A relay regulator, having constant parameters and no underlying plant model, performs intensive vehicle maneuvering at snow, ice and mixed surface with good path tracking accuracy. The relay regulator with second order integration of the output signal operates with chattering, which most likely would be unacceptable in actual implementations. Increasing of the regulator order (both input derivative and output integration) by one allows to eliminate chattering. Simulation results show that despite high integration order the regulator operation is stable, while the tracking accuracy degrades negligibly. However, increasing of the regulator's order questions the calculation quality of derivatives, since some noise is inevitable in feedback signals. In this regard, the usually claimed robustness of sliding modes against disturbances becomes a crucial feature to be the subject of further research.

\section{Acknowledgments}

The article was prepared under the agreement \#14.625.21.0043 with the Ministry of Education and Science of the Russian Federation (unique project identifier RFMEF162517X0043).

\section{References}

1. M. Alirezaei, S.T.H. Jansen, A.J.C. Schmeitz, A.K Madhusudhanan, Collision Avoidance System using State Dependent Riccati Equation Technique: An Experimental Robustness Evaluation, 13th Int. Symposium on Advanced Vehicle Control (AVEC'16), pp. 127-132 (2016).

2. T. Keviczky, P. Falcone, F. Borrelli, J. Asgari, D. Hrovat, Predictive control approach to autonomous vehicle steering, American Control Conference, pp. 6-15 (2006), doi: 10.1109/ACC.2006.1657458.

3. M. Keller, C. Haß, A. Seewald, T. Bertram, A vehicle lateral control approach for collision avoidance by emergency steering maneuvers, 6th Int. Munich Chassis Symposium, pp. 175-197 (2015), doi: 10.1007/978-3-65809711-0_15.

4. P. Zhă, J. Chen, Y. Song, X. Tao, T. Xu, T. Mei, Design of a Control System for an Autonomous Vehicle Based on Adaptive-PID, Int. Journal of Advanced Robotic Systems 9(2), 1 (2012), doi: 10.5772/51314

5. N.C. Basjaruddin, Kuspriyanto, Suhendar, D. Saefudin, S.A. Aryani, Lane Keeping Assist System Based on Fuzzy Logic, Int. Electronics Symposium (IES), pp. 116-119 (2015), doi: 10.1109/ELECSYM.2015.7380824

6. J. Pérez, V. Milanés, E. Onieva, Cascade Architecture for Lateral Control in Autonomous Vehicles, IEEE Transactions on Intelligent Transportation Systems,12(1), pp. 73-82 (2011), doi: 10.1109/TITS.2010.2060722.

7. S.V. Emelyanov, Automatic Control Systems with Variable Structure (in Russian), Moscow, "Nauka" (1967).

8. V. Utkin, J. Guldner, J. Shi, Sliding Mode Control in Electromechanical Systems, Second Edition, Taylor \& Francis Group, LLC (2009).

9. A. Levant, Chattering Analysis, IEEE Transactions on Automatic Control, 55(6), pp. 1380-1389 (2010), doi: 10.1109/TAC.2010.2041973.

10. J. J. Slotine, S. S. Sastry, Tracking control of nonlinear systems using sliding surfaces, with application to robot manipulators, International Journal of Control, 38(2), pp. 465-492 (1983), doi:10.1080/00207178308933088. 
11. L. Fridman, A. Levant, Higher-Order Sliding Modes, book chapter (Sliding Mode Control in Engineering, chapter 3), Inc. Marcel Dekker (2002).

12. A. Ferrara, Sliding mode control of vehicle dynamics, London: Institution of Engineering and Technology (2017), doi:10.1049/PBTR005E.

13. G. Tagne, R. Talj, A. Charara, Higher-Order Sliding Mode Control for Lateral Dynamics of Autonomous
Vehicles, with Experimental Validation, IEEE Intelligent Vehicles Symposium (IV) (2013), doi: 10.1109/IVS.2013.6629545. 14. I.A. Kulikov, S.V. Bakhmutov, A.A. Barashkov, An Investigation of Vehicle Dynamics Concerning Active Safety Systems by Simulations and Driving Tests, Proceedings of NAMI, 265, pp. 53-65 (2016).

15. H.B. Pacejka, I. Besselink, Tire and vehicle dynamics, Third Edition, Elsevier Ltd., pp. 165-183 (2012). 